\title{
Contemporary treatment of anxiety in primary care: a systematic review and meta-analysis of outcomes in countries with universal healthcare
}

\author{
Erin L. Parker ${ }^{1 *}$, Michelle Banfield ${ }^{2}$, Daniel B. Fassnacht ${ }^{1,3}$, Timothy Hatfield ${ }^{1}$ and Michael Kyrios ${ }^{3}$
}

\begin{abstract}
Background: Anxiety disorders are highly prevalent mental health conditions and are managed predominantly in primary care. We conducted a systematic review and meta-analysis of psychological and pharmacological treatments in countries with universal healthcare, and investigated the influence of treatment provider on the efficacy of psychological treatment.
\end{abstract}

Method: PubMed, Cochrane, PsycINFO, CINAHL, and Scopus were searched in April 2017 for controlled studies of evidence-based anxiety treatment in adults in primary care, published in English since 1997. Searches were repeated in April 2020. We synthesised results using a combination of meta-analysis and narrative methods. Meta-analysis was conducted using a random-effects multi-level model to account for intercorrelation between effects contributed different treatment arms of the same study. Moderator variables were explored using meta-regression analyses.

Results: In total, 19 articles (from an initial 2,247) reporting 18 studies were included. Meta-analysis including ten studies $(n=1,308)$ found a pooled effect size of $g=1.16(95 \% \mathrm{Cl}=0.63-1.69)$ for psychological treatment compared to waitlist control, and no significant effect compared to care as usual ( $p=.225)$. Substantial heterogeneity was present $\left(I^{2}=81.25\right)$. Specialist treatment produced large effects compared to both waitlist control $(g=1.46,95 \% \mathrm{Cl}=0.96$ $-1.96)$ and care as usual $(g=0.76,95 \% \mathrm{Cl}=0.27-1.25)$. Treatment provided by non-specialists was only superior to waitlist control $(g=0.80,95 \% \mathrm{Cl}=0.31-1.28)$. We identified relatively few studies $(n=4)$ of medications, which reported small to moderate effects for SSRI/SNRI medications and hydroxyzine. The quality of included studies was variable and most studies had at least "unclear" risk of bias in one or more key domains.

Conclusions: Psychological treatments for anxiety are effective in primary care and are more effective when provided by a specialist (psychologist or clinical psychologist) than a non-specialist (GP, nurse, trainee). However, non-specialists provide effective treatment compared with no care at all. Limited research into the efficacy of pharmacological treatments in primary care needs to be considered carefully by prescribers

Trial registration: PROSPERO registration number CRD42018050659

Keywords: Anxiety, Systematic review, Meta-analysis, Psychological treatment, Pharmacological treatment, Primary care

\footnotetext{
*Correspondence: erin.parker@anu.edu.au

1 Research School of Psychology, Australian National University, Canberra,

ACT 2601, Australia

Full list of author information is available at the end of the article
}

(C) The Author(s) 2021. Open Access This article is licensed under a Creative Commons Attribution 4.0 International License, which permits use, sharing, adaptation, distribution and reproduction in any medium or format, as long as you give appropriate credit to the original author(s) and the source, provide a link to the Creative Commons licence, and indicate if changes were made. The images or other third party material in this article are included in the article's Creative Commons licence, unless indicated otherwise in a credit line to the material. If material is not included in the article's Creative Commons licence and your intended use is not permitted by statutory regulation or exceeds the permitted use, you will need to obtain permission directly from the copyright holder. To view a copy of this licence, visit http://creativecommons.org/licenses/by/4.0/. The Creative Commons Public Domain Dedication waiver (http://creativeco mmons.org/publicdomain/zero/1.0/) applies to the data made available in this article, unless otherwise stated in a credit line to the data. 


\section{Background}

Anxiety disorders are among the most prevalent mental health conditions globally, affecting approximately one in nine people in a given year [1]. These conditions are associated with substantial impairments in occupational and social functioning, including unemployment and under-employment, social isolation, and interpersonal and marital conflict [2]. Anxiety disorders are a leading cause of disability, accounting for more years lived with a disability than any other mental health condition, as well as many physical health conditions [3].

Anxiety disorders are managed predominantly within primary care and are one of the most common conditions seen in these settings, despite less than half of those with an anxiety disorder seeking help [4-6]. Treating anxiety in primary care has substantial advantages in terms of ease of access and financial cost. Indeed, integrating mental health services in primary care is considered a key component of achieving universal health coverage [7]. However, only a minority of people seeking help in primary care receive adequate treatment for their anxiety $[8,9]$. Anxiety disorders tend to have a chronic course if insufficiently treated, resulting in significant impairment for the individual and high economic costs due to repeat service use and decreased work productivity $[3,10]$. Furthermore, delayed or inadequate treatment increases the likelihood of developing common co-occurring conditions such as depression and substance use, which are associated with greater impairment [10].

Several different professionals may provide treatment for anxiety disorders in primary care (e.g., social workers, nurses, psychologists), though the majority of treatment is provided by general practitioners (GPs) $[6,11]$. Best practice treatment involves a stepped-care approach based on severity of symptoms and functional impairment, as well as consideration of co-occurring difficulties, consumer preferences, and previous treatment [12, 13]. The specific steps vary by disorder, and include low intensity psychological interventions (e.g., guided or unguided self-help, psychoeducation groups) for milder or uncomplicated anxiety problems, and higher-intensity treatments such as individual cognitive behavioural therapy (CBT) or medications for more moderate problems, or where low-intensity interventions have been unsuccessful $[14,15]$. For complex and severe anxiety difficulties, referral to specialist mental health services outside of primary care should be considered [14, 15]. In general, psychological interventions are recommended as first line in preference to pharmacological treatment [12]. However, pharmacological interventions are the most common treatment provided in primary care regardless of anxiety severity $[8,11]$, and despite research suggesting consumers prefer psychological therapies $[16,17]$.
Although GPs are not routinely able to provide highintensity psychological treatments due to limited training and time pressures $[18,19]$, they can offer low intensity interventions such as psychoeducation and self-help programs. In particular, computerised or internet-delivered CBT has been shown to be effective for treating anxiety, and may be as effective as face-to-face CBT [20, 21]. Computerised CBT programs usually involve modules delivered by desktop, internet, or phone applications, and are suitable for provision in primary care as either guided (i.e., with support from a clinician) or unguided interventions [20].

When appropriate, higher intensity therapies can such as face-to-face CBT can also be provided in primary care by other lay providers (e.g., nurses), which has been a focus of recent research to improve access to these therapies [22]. However, financing of non-specialists to deliver psychosocial interventions remains a barrier in many countries, and may explain why GPs continue to provide the majority of care for anxiety disorders. In addition, while there is emerging evidence for psychological interventions provided by non-specialists, the majority of outcome research involves treatment provided by mental health specialists. For example, a previous systematic review and meta-analysis of psychological treatment in primary care found a moderate effect size for reducing anxiety symptoms [23]. However, the treatment in most included studies was provided by clinical psychologists, who do not typically work in primary care settings.

Medications such as selective serotonin reuptake inhibitors (SSRIs) or serotonin noradrenaline reuptake inhibitors (SNRIs) are also recommended treatments for anxiety $[12,13]$ and may be cheaper and more accessible to consumers than psychological treatments. However, their effectiveness when prescribed in primary care populations, and without any combined psychological management, is unclear. Benzodiazepine medications also remain frequently prescribed for anxiety despite not being a current recommended treatment $[24,25]$. To our knowledge, no previous reviews of pharmacological anxiety interventions in primary care exist.

In this review, we aimed to synthesise contemporary evidence for the effect of psychological and pharmacological treatments for anxiety compared with control in primary care. We were interested in evidence from studies that most accurately reflected the real-world treatment settings in which they were conducted. To this end, we focused on reviewing evidence from countries with existing universal healthcare systems (i.e., where mental health services are routinely provided in primary care without significant cost to consumers). Regarding psychological treatments, our review sought to update and extend upon the review conducted by Seekles et al. 
[17] by a) maximising identification of studies where treatment was provided by non-specialists or GPs, and b) excluding studies of obsessive compulsive disorder (OCD) and post-traumatic stress disorder (PTSD), which are no longer considered anxiety disorders in the most recent classification systems. We also sought to investigate variables that may moderate psychological treatment effectiveness, namely treatment provider (specialist vs. non-specialist) and treatment modality (face-to-face vs. online vs. self-help).

\section{Method}

\section{Search strategy and selection process}

This review followed Preferred Reporting Items for Systematic Reviews and Meta-Analyses (PRISMA) guidelines and was registered with the international prospective register of systematic reviews (PROSPERO; registration number CRD42018050659). Primary searching was conducted in PubMed using MeSH terms (see Table 1). PsycINFO, the Cochrane Central Register of Controlled Trials (CENTRAL), the Cumulative Index to Nursing and Allied Health Literature (CINAHL), and Scopus were also searched to maximise identification of relevant studies. The full search strategy for all databases is available in additional file 1.

We identified and removed duplicate articles using Endnote Referencing software. Two independent researchers (ELP and TH) screened titles and abstracts of retrieved articles to determine eligibility for the review. ELP and TH then screened full-text versions of all eligible studies for final inclusion. The reference lists of included articles were hand-searched to identify additional studies, and none were found. Disagreements between reviewers were resolved through post-assessment discussion at each stage of the process.

Initial searches were conducted on April 17, 2017. We re-ran searches on 22 April 2020 to identify any studies published in the period since our initial search date. The first author screened the additional records retrieved following the same process as above. Our inclusion and exclusion criteria can be seen in Table 2.

We were interested in synthesising the most recent evidence for treating anxiety in primary care. As such, we excluded studies published prior to 1997, which was 20 years before our initial search. We included studies of participants with a primary diagnosis of an anxiety disorder according to diagnostic criteria (DSM or ICD), or clinically significant levels of anxiety on an assessment/ screening measure (e.g., Beck Anxiety Inventory [BAI]; Depression Anxiety Stress Scales [DASS]). We excluded studies of OCD and PTSD, which are no longer classified as anxiety disorders. Studies focusing on mixed anxiety/depression were included due to the high rates of co-occurrence between these conditions, as long as treatment was anxiety-specific (i.e., recommended pharmacological agents for anxiety, or anxiety-focussed psychological treatment).

We defined evidence-based treatments as psychological and pharmacological interventions with an existing evidence base, as determined by current clinical practice guidelines (e.g., NICE guidelines, [12]). For psychological interventions, this included self-help, mindfulness/ applied relaxation, and individual cognitive behavioural therapy [12, 14, 15]. Pharmacological treatments included SSRIs, SNRIs, pregabalin (generalised anxiety disorder), tricyclic antidepressants (panic disorder) and benzodiazepines in the case of short-term treatment [12, $14,15]$.

\section{Data extraction and synthesis}

The primary outcome in this review was treatment effect size (standardised mean difference) for the reduction of anxiety symptoms in each study. Secondary outcomes were treatment effect sizes for reduction in depressive symptoms and improvement in quality of life. Included papers were coded by two independent reviewers (ELP and either $\mathrm{TH}$ or DBF) using a standardised data extraction form. We extracted the following variables from each study: demographic information about participants

Table 1 MeSH terms used for primary searching in PubMed

\begin{tabular}{lc}
\hline Topic & MeSH terms \\
\hline Anxiety & "Anxiety Disorders" OR "Anxiety" \\
Primary Care & "Primary Health Care" OR "Physicians, Primary Care" OR "General Practice" OR \\
& "General Practitioners" OR "Physicians, Family" OR "Primary Care Nursing" \\
& OR "Family Nursing" OR "Nurses, Community Health" OR "Nurse Practition- \\
ers" OR "Nurse Clinicians" & "Outcome Assessment (Health Care)" \\
Treatment (general) & "Psychotherapy" OR "Counseling" OR "Relaxation" \\
Treatment (psychological) & "Drug Therapy" OR "Psychotropic Drugs" OR "Adrenergic beta-Antagonists" \\
\hline
\end{tabular}


Table 2 Inclusion and exclusion criteria

\begin{tabular}{|c|c|c|}
\hline & Inclusion criteria & Exclusion criteria \\
\hline Publication details & $\begin{array}{l}\text { Peer-reviewed journal articles reporting primary data } \\
\text { Published since } 1997 \\
\text { Article written in English }\end{array}$ & $\begin{array}{l}\text { Published before } 1997 \\
\text { Secondary data analysis, literature reviews, meta-analyses }\end{array}$ \\
\hline Study type & Controlled trials & Uncontrolled trials \\
\hline Population & $\begin{array}{l}\text { Adults ( } 18+\text { years) } \\
\text { Primary diagnosis of anxiety disorder or clinically significant } \\
\text { anxiety } \\
\text { Mixed anxiety/depression }\end{array}$ & $\begin{array}{l}\text { Persons under } 18 \text { years } \\
\text { Primary diagnosis of other mental health condition (e.g., depres- } \\
\text { sion, OCD, PTSD) }\end{array}$ \\
\hline Setting & $\begin{array}{l}\text { Primary care } \\
\text { Country with universal healthcare }\end{array}$ & Secondary or tertiary care setting (e.g., hospital, psychiatric clinic) \\
\hline Treatment & $\begin{array}{l}\text { Evidence-based psychological or pharmacological treatments } \\
\text { for anxiety }\end{array}$ & $\begin{array}{l}\text { Alternative treatments (e.g., kava) } \\
\text { Treatment focusing on condition other than anxiety (e.g., CBT for } \\
\text { depression) }\end{array}$ \\
\hline Outcome & At least one measure of anxiety symptomatology & No measure of anxiety symptoms included \\
\hline
\end{tabular}

(age, gender); country in which the study was conducted; type of anxiety; treatment type; modality of treatment (e.g., self-help, online, face-to-face); treatment provider; type of control group; and outcome statistics (means and standard deviations between groups at post-treatment and follow-up, or other statistics where these were not available). Data were extracted from published reports, and study authors were contacted to obtain missing information. We assessed interrater agreement by comparing the information on each reviewer's coding form after extraction of all items. Disagreements were resolved through discussion and review of the information in the article.

We calculated standardised mean differences (Hedges g) [26] and standard errors at post-treatment between control and treatment groups for each study. This was calculated from means and standard deviations or other statistics (e.g., t-value, p-value) when the former were not reported. Hedge's g was chosen over other measures of effect size as it corrects for small sample sizes [27], which was an issue for some of the studies in this review. We calculated a separate effect size for all active treatments compared with control in studies with multiple treatment arms. If an anxiety-specific measure was not the primary outcome in the study, the best (e.g., gold standard for a particular disorder, best test-retest reliability) measure of anxiety symptoms in the study was chosen to calculate these statistics. Measures from each study are reported in Table 3.

Meta-analysis was performed on studies of psychological treatment only, and other studies were synthesised using narrative methods. We conducted meta-analysis in RStudio version 1.0.143 using the metafor package [28]. For studies with multiple treatment arms, we entered effect sizes from each active treatment compared with the control group into this analysis. A random-effects multi-level model was used to account for intercorrelation between effect sizes contributed by the same study, and meta-regression analyses were run to investigate the effects of moderator variables. We obtained the code for these analyses from the metafor package website (www.metafor-project.org) based on the description of meta-analysis for multiple treatment studies [29] and multivariate random and mixed-effects models [30]. We assessed variability between studies using $\mathrm{Chi}^{2}$ tests and $\mathrm{I}^{2}$ estimates of heterogeneity. Interpretation of $\mathrm{I}^{2}$ values was based on guidelines from the Cochrane handbook, where $0 \%$ to $40 \%$ represents heterogeneity that may not be important; $30 \%$ to $60 \%$ may represent moderate heterogeneity; $50 \%$ to $90 \%$ may represent substantial heterogeneity; and $75 \%$ to $100 \%$ represents considerable heterogeneity [31]. Heterogeneity was explored using meta-regression to investigate the effect of moderators, as noted above.

Publication bias was investigated with Egger's regression test of funnel plot asymmetry [32, 33] by using sampling variance as a moderator in a multi-level model. Methods of sensitivity analysis are not yet well developed for multivariate/multi-level models [34], and options (e.g., Trim and Fill) are not currently available in the metafor package for these types of models. Therefore, we conducted sensitivity analysis by calculating Cook's distance $[35,36]$ to identify influential outliers. These were defined as observations with a Cook's distance greater than $4 / n$.

\section{Risk of bias}

Risk of bias for each study was assessed by ELP and DBF independently using the Cochrane Collaboration's risk of bias tool [37]. In many psychological treatment studies, blinding of participants and personnel is not possible due to the interpersonal nature of the treatment. 
Table 3 Characteristics of included studies

\begin{tabular}{|c|c|c|c|c|c|c|c|c|c|}
\hline First Author, Year & Country & $\mathrm{n}$ & FU & Disorder & Outcome & Treatment & Modality & Provider & Control \\
\hline \multicolumn{10}{|c|}{ Psychological Treatment Studies } \\
\hline Berger, 2017 & $\begin{array}{l}\text { Germany/Switzer- } \\
\text { land/Austria }\end{array}$ & 139 & 6-mth & Anx & $\mathrm{BAl}$ & CBT & Online & Self & CAU \\
\hline Gensichen, 2019 & Germany & 419 & 6-mth & Anx & $\mathrm{BAl}$ & CBT & $\begin{array}{l}\text { Guided biblio- } \\
\text { therapy }\end{array}$ & GP & CAU \\
\hline Kendrick, 2005 (1) & United Kingdom & 247 & 4-mth & CMD & HADS-A & Other & $\mathrm{F} 2 \mathrm{~F}$ & Mental health nurse & CAU \\
\hline Kendrick, 2005 (2) & & & & & & Other & $\mathrm{F} 2 \mathrm{~F}$ & Mental health nurse & CAU \\
\hline Klein, 2006 (1) & Australia & 55 & 3-mth & Anx & PDSS & CBT & Online & Psychologist & Waitlist \\
\hline Klein, 2006 (2) & & & & & & CBT & Bibliotherapy & Trainee psychologist & Waitlist \\
\hline Newby, 2013 & Australia & 99 & 3-mth & CMD & GAD-7 & CBT & Online & Unspecified clinician & Waitlist \\
\hline Nordgren, 2014 & Sweden & 100 & 10-mth & Anx & BAl & CBT & Online & Trainee psychologist & Waitlist \\
\hline Power, 2000 (1) & Scotland & 104 & 6-mth & Anx & HAM-A & CBT & $\begin{array}{l}\text { Guided (std.) biblio- } \\
\text { therapy }\end{array}$ & Clinical psychologist & CAU \\
\hline Power, 2000 (2) & & & & & & CBT & $\begin{array}{l}\text { Guided (min.) biblio- } \\
\text { therapy }\end{array}$ & Clinical psychologist & CAU \\
\hline Seekles, $2011 \mathrm{a}$ & Netherlands & 108 & - & Anx & HADS-A & Other/CBT & $\begin{array}{l}\text { Guided online/bib- } \\
\text { liotherapy }\end{array}$ & Mental health nurse & $C A U$ \\
\hline Sharp, 2004 (1) & United Kingdom & 97 & 3 -mth & Anx & HAM-A & CBT & $\mathrm{F} 2 \mathrm{~F}$ & Clinical psychologist & Waitlist \\
\hline Sharp, 2004 (2) & & & & & & CBT & F2F - group & Clinical psychologist & Waitlist \\
\hline Sundquist, 2015 & Sweden & 215 & - & CMD & HADS-A & Other & $F 2 F-$ group & $\begin{array}{l}\text { Psychologist/coun- } \\
\text { sellor }\end{array}$ & CAU \\
\hline van Boeijen, 2005 & Netherlands & 142 & 10-mth & Anx & STAI-S & CBT & $\begin{array}{l}\text { Guided biblio- } \\
\text { therapy }\end{array}$ & GP & CAU \\
\hline \multicolumn{10}{|c|}{ Pharmacological Treatment Studies } \\
\hline $\begin{array}{l}\text { Laakmann, } 1998 \\
\text { (1) }\end{array}$ & Germany & 125 & - & Anx & HAM-A & Buspirone & Tablet & GP & Placebo \\
\hline $\begin{array}{l}\text { Laakmann, } 1998 \\
\text { (2) }\end{array}$ & & & & & & Lorazepam & Tablet & GP & Placebo \\
\hline Lader, 1998 (1) & $\begin{array}{l}\text { France and United } \\
\text { Kingdom }\end{array}$ & 244 & - & Anx & HAM-A & Hydroxyzine & Tablet & GP & Placebo \\
\hline Lader, 1998 (2) & & & & & & Buspirone & Tablet & GP & Placebo \\
\hline Lenox-Smith, 2003 & United Kingdom & 244 & - & Anx & HAM-A & Venlafaxine & Tablet & GP & Placebo \\
\hline Llorca, 2002 (1) & France & 334 & - & Anx & HAM-A & Hydroxyzine & Tablet & GP & Placebo \\
\hline Llorca, 2002 (2) & & & & & & Bromazepam & Tablet & GP & Placebo \\
\hline \multicolumn{10}{|c|}{ Combined Treatment and Stepped Care Studies } \\
\hline Blomhoff, 2001 (1) & United Kingdom & 387 & - & Anx & SPS & Sertraline + CBT & $\mathrm{F} 2 \mathrm{~F}+$ tablet & GP & Placebo \\
\hline Blomhoff, 2001 (2) & & & & & & Sertraline & Tablet & GP & Placebo \\
\hline Blomhoff, 2001 (3) & & & & & & CBT & $\mathrm{F} 2 \mathrm{~F}$ & GP & Placebo \\
\hline Muntingh, 2014 & Netherlands & 180 & 9-mth & Anx & BAl & Stepped Care & Multiple & Multiple & CAU \\
\hline Oosterbaan, 2013 & Netherlands & 158 & 4-mth & CMD & HAM-A & Stepped Care & Multiple & Multiple & CAU \\
\hline Seekles, $2011 \mathrm{~b}$ & Netherlands & 120 & - & CMD & HADS-A & Stepped Care & Multiple & Multiple & $C A U$ \\
\hline
\end{tabular}

Anx anxiety disorders only, CMD common mental disorders, BAI Beck Anxiety Inventory, GAD-7 Generalized Anxiety Disorder 7-item Scale, HADS-A Hospital Anxiety and Depression Scale-Anxiety Subscale, HAM-A Hamilton Anxiety Scale, PDSS Panic Disorder Severity Scale, SPS Social Phobia Scale, STAI-S State Trait Anxiety Inventory-State Subscale, CBT Cognitive Behaviour Therapy, F2F face-to-face therapy, GP general practitioner, CAU care as usual, FU follow-up length post-treatment, $n$ total $\mathrm{n}$ for study

In these cases, we rated studies as having "unclear" risk of bias for this criterion, providing no other factors warranted a rating of "high". Consistent with similar reviews of heterogeneous studies with complex interventions [38], we sought agreement between reviewers for all items by comparing ratings and resolved disagreements through post-assessment discussion.

\section{Results}

\section{Description of studies}

Our initial search identified 2,151 articles (after removal of duplicates), and 207 full-text articles were screened. Eighteen articles reporting 17 studies met all inclusion criteria. Interrater agreement for extracted variables was 89.3\%. Updated searching in April 2020 
identified only one further study for inclusion (from an initial 95 articles published since our original search). Of the 191 articles excluded after full-text screening, 71 were excluded on the basis of being conducted in a country without universal healthcare (all from the USA). Thirty-one of these articles were publications from a single, large study of collaborative care for anxiety [39]. The full study selection process can be seen in Fig. 1.
A total of 19 articles reporting 18 studies met all criteria and were included in our review. Two articles reported separate steps of the same study [40, 41], and eight studies involved more than one active treatment condition [19, 42-49]. Across all studies, there were 28 comparisons of active treatment with a control group (placebo, waitlist control, or care as usual [CAU]). Key characteristics of the included studies are available in Table 3.

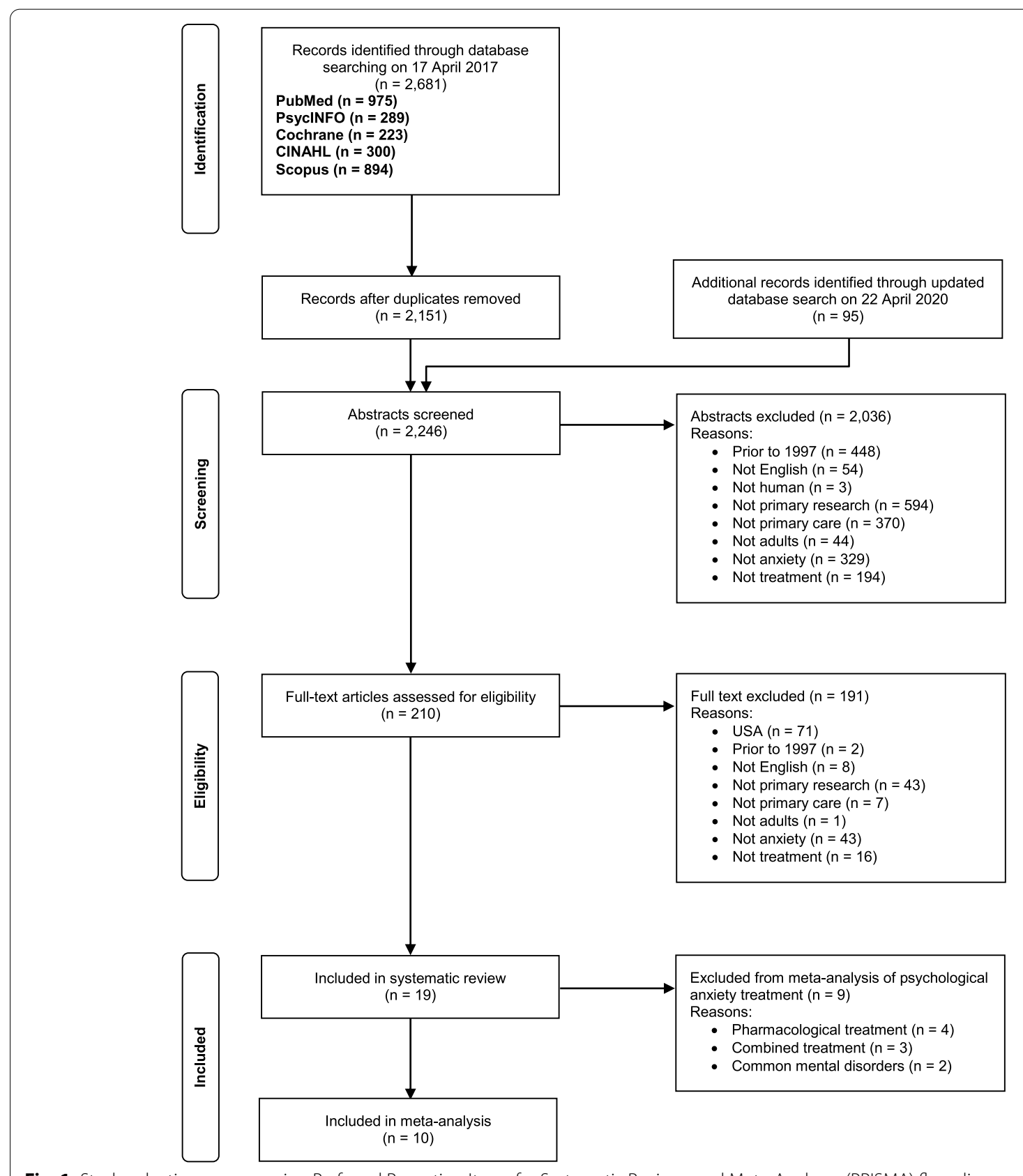

Fig. 1 Study selection process using Preferred Reporting Items for Systematic Reviews and Meta-Analyses (PRISMA) flow diagram 


\section{Participants}

In the included studies, 2,059 participants were randomised to an active treatment condition and 1,247 to a control condition. Participants ranged in age from 18 to 80 years, with the average age in each study between 34.2 years and 51 years. All studies had a higher proportion of women than men.

Thirteen studies investigated anxiety disorders specifically; four generalised anxiety disorder (22.2\% of 18$)$, four panic disorder with or without agoraphobia (22.2\% of 18 ), and five investigated multiple anxiety disorders (including mixed anxiety/depression; $27.8 \%$ of 18 ). Five studies (27.8\% of 18 studies) included participants with "common mental disorders" as their primary diagnosis, which referred to one or more of anxiety disorders, depression, mixed anxiety/depression, and stress/adjustment disorders. One study reported separate outcomes for participants with an anxiety disorder only [40] and anxiety-only data was obtained from the authors for another study [43].

Most studies reported moderate mean anxiety severity at baseline among participants, as measured by either clinician (e.g., CGI-S, HAM-A) or self-report (e.g., BAI) measures. Two studies reported mild-to-moderate anxiety severity at baseline $[41,43]$, and five studies reported moderate-severe or severe anxiety $[19,44,45,50,51]$.

\section{Treatment and control group type}

The majority of included studies were of psychological treatments $(10 / 18,55.5 \%)$. Four studies investigated one or more pharmacological treatments (22.2\% of 18$)$, and one study compared psychological and pharmacological treatments (and their combination). The remaining three studies investigated the effect of stepped care, which included both psychological and pharmacological treatments. Pharmacological studies tended to be older (published between 1998 and 2003) than psychological studies (published between 2000 and 2019).

In the 10 psychological treatment studies, four compared treatment with a waitlist control (i.e., no treatment) and six used a CAU control. The care received by control group participants was described in four of the six CAU-controlled studies [19, 48, 50, 52], and most commonly included antidepressants, benzodiazepines, CBT, or referral for specialist mental health care. These studies reported that most control group participants received at least one of these treatments, though did not report actual numbers for the different types of care, with the exception of one study [50]. All three studies of stepped care used CAU as a control and provided descriptions of the care received by participants. At least half of control group participants in these studies received medication (antidepressants or benzodiazepines), referral to a specialist mental health professional, or both. All pharmacological treatment studies used placebo controls.

\section{Psychological interventions}

Four psychological treatment studies investigated the effects of two different treatments with a control. With the addition of the psychological treatment arm from the study of combined treatment [42] as well as the article reporting outcomes for the self-help step [40] of a stepped care study [41], there were a total of 16 comparisons of psychological treatment with either CAU or waitlist control.

Psychological treatments were predominantly CBTbased ( $n=13,81.2 \%$ of 16$)$ and provided on an individual basis. One study involved group treatment [52], and one study compared individual treatment with group treatment [49]. Treatment was delivered either face-to-face with a health professional $(n=6,37.5 \%$ of 16$)$ or through self-help manuals/internet programs with support from a professional $(n=10,62.5 \%$ of 16$)$. Treatment was provided by specialists (clinical psychologists or psychologists) in six treatment conditions (37.5\% of 16$)$. In the other ten treatment conditions, treatment was provided by trainee psychologists $(n=2)$, mental health nurses $(n=3)$, GPs $(n=3)$, an unspecified clinician $(n=1)$, and the participant themselves $(n=1)$, all of whom we coded as non-specialists in this review.

\section{Effect on anxiety disorders}

We conducted meta-analysis on the studies of psychological treatment for anxiety disorders; to limit heterogeneity, we excluded the studies of common mental disorders and mixed anxiety/depression from this analysis [43, 53]. The effect of psychological treatment on common mental disorders is instead described below using narrative synthesis. Meta-analysis included 14 comparisons of psychological treatment with a control group, taken from ten studies (Fig. 2, Table 4). The model found a large effect size for psychological treatment compared to waitlist control $(g=1.16,95 \% \mathrm{CI}=0.63-1.69)$, and no significant effect compared to CAU control $(\mathrm{Z}=1.21, p=0.225)$. Considerable heterogeneity was present $\left(\mathrm{I}^{2}=81.25\right)$.

Due to a lack of power, we were only able to investigate the effects of one moderator variable. Treatment provider was chosen as this variable was more relevant to the aims of the review. Meta-regression analysis found that treatment effect was significantly moderated by treatment provider $(\mathrm{z}=2.61, p=0.009)$. Results are presented in Table 4. The inclusion of this moderator accounted for $53 \%$ of the total amount of heterogeneity. However, the resulting test for residual heterogeneity was significant $\left(\mathrm{Q}_{\mathrm{E}}=36.22, \mathrm{df}=11, p<0.001\right)$. 


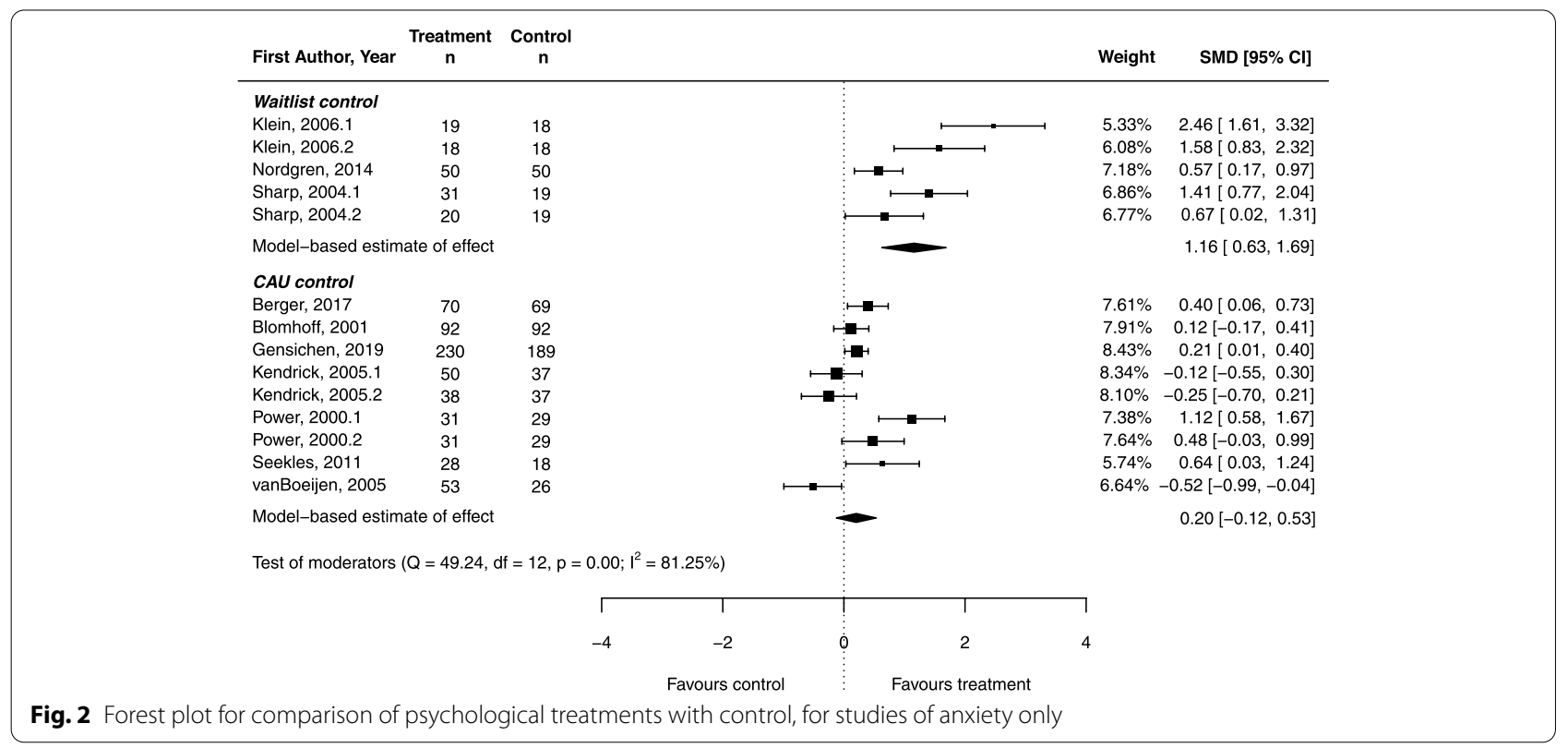

Treatment provided by a non-specialist compared with CAU did not produce a significant effect on anxiety symptoms $(p=0.468)$. However, compared with waitlist control a large effect was found $(g=0.80,95 \% \mathrm{CI}=0.31$ 1.28 ). Treatment provided by a specialist was associated with large effects regardless of the comparison group (CAU: $g=0.76,95 \% \mathrm{CI}=0.27-1.25$; waitlist: $g=1.46$, $95 \% \mathrm{CI}=0.96-1.96)$.

Egger's regression test showed significant funnel plot asymmetry $(\mathrm{z}=3.70, p<0.001)$, indicating the presence of publication bias. No influential outliers were identified, though Cook's distance for one study [19] was substantially larger $(D=0.23)$ than for other studies and close to the threshold of $0.29(4 / \mathrm{n})$, suggesting this study had a larger influence on the model than the other observations.

\section{Effect on common mental disorders}

One study investigated two types of psychological treatment (problem-solving and generic mental health nurse care) for common mental disorders (anxiety, depressive, stress, and adjustment disorders) and found no significant treatment effect for either compared with CAU [43]. The authors for this study also provided us with results for participants with anxiety only, which are reported in the meta-analysis above. A second study investigated online CBT for mixed anxiety and depression and found a large effect size of $g=0.85(95 \% \mathrm{CI}=0.43-1.27)$ compared with waitlist control [53].

\section{Pharmacological interventions}

All four pharmacological studies investigated medications for generalised anxiety disorder (GAD), with three examining the relative efficacy of two different medications. There were a total of eight comparisons of pharmacological treatment with placebo, including the pharmacological treatment arm of the study of combined treatment (which studied generalised social phobia) [42]. Meta-analysis was not possible for these comparisons due to incomplete reporting of outcome statistics in the primary articles.

Two comparisons of benzodiazepines with placebo $[45,47]$ found no significant difference between groups at post-treatment. Authors in two studies [45, 46] also reported no effect of buspirone compared with placebo. Both studies comparing hydroxyzine with placebo found a significant treatment effect; one reported a moderate effect size of $g=0.47(95 \% \mathrm{CI}=0.16-0.78)$ at posttreatment [46], and the other found a similar effect size of $g=0.32(95 \% \mathrm{CI}=0.05-0.60)$ [47]. Likewise, both studies of SSRI/SNRI medications reported a treatment effect, with small effects of $g=0.29$ (95\% CI $=0.00-0.58)$ found for sertraline compared with placebo [42], and $g=0.25(95 \% \mathrm{CI}=0.00-0.50)$ for venlafaxine compared with placebo [51].

\section{Combined interventions}

We did not perform meta-analysis on studies of combined interventions due to the small number of studies and the clinical diversity among them. The sole study of combined psychological and pharmacological treatment investigated the relative effects of exposure therapy, sertraline, and exposure therapy plus sertraline compared with placebo [42]. The results for psychological treatment and pharmacological treatment in this study have 
Table 4 Meta-analytic results for effect of psychological treatment on anxiety symptoms

\begin{tabular}{|c|c|c|c|c|c|c|}
\hline & $n$ & $g$ & se & $95 \% \mathrm{Cl}$ & $z$ & $p$ \\
\hline All studies & 14 & 0.49 & 0.20 & $0.10-0.88$ & 2.44 & .015 \\
\hline Treatment vs. CAU & 9 & 0.20 & 0.17 & $-0.12-0.53$ & 1.21 & .225 \\
\hline Treatment vs. waitlist & 5 & 1.16 & 0.27 & $0.63-1.69$ & 4.28 & $<.0001$ \\
\hline Non-specialist provider & 9 & & & & & \\
\hline CAU control & 7 & 0.10 & 0.13 & $-0.16-0.35$ & 0.73 & .468 \\
\hline Waitlist control & 2 & 0.80 & 0.25 & $0.31-1.28$ & 3.22 & .001 \\
\hline Specialist provider & 5 & & & & & \\
\hline CAU control & 2 & 0.76 & 0.25 & $0.27-1.25$ & 3.04 & .002 \\
\hline Waitlist control & 3 & 1.46 & 0.26 & $0.96-1.96$ & 5.71 & $<.001$ \\
\hline
\end{tabular}

$n$ number of comparisons in analysis, se standard error, $C A U$ care as usual

been reported above. A significant treatment effect was also found for combined treatment compared with control, with an effect size of $g=0.35(95 \% \mathrm{CI}=0.07-0.64)$. Although combined treatment produced the largest effect size, this was not significantly different from the other active treatment groups.

In the three studies of stepped care $[41,54,55]$, treatment was provided by multiple professionals, including mental health nurses and psychiatrists. Higher and more intensive steps of these interventions included medication combined with psychological therapy. Two studies found small, significant effects of stepped care compared to CAU for common mental disorders $(g=0.23,95 \% \mathrm{CI}=-0.13-0.58 \quad[41] ; g=0.31$, $95 \% \mathrm{CI}=-0.01-0.63[55])$. The third study investigated stepped care for anxiety only, and also found a significant effect $(g=0.21,95 \% \mathrm{CI}=-0.12-0.54)$ [54].

\section{Longer-term follow-up}

Follow-up of at least three months post-treatment was reported in 11 of the 18 included studies. Outcomes were difficult to synthesise due to variability in how these statistics were reported and are described below using narrative methods.

All but one of the psychological treatment studies [52] reported follow-up data. For studies where a waitlist control was used, three studies reported maintenance of gains within the treatment group at three-[44, 53] and 10-month [56] follow up. Control group data was not recorded in these studies as control participants received the intervention after the waiting period. A fourth study, which investigated the effect of group and individual CBT, reported gains in the group CBT condition were maintained at follow-up, but the rate of clinically significant change decreased in the individual CBT condition [49].

Among studies comparing to a CAU control, four reported outcomes for both control and treatment groups at follow-up. There was no significant difference between treatment and control groups in two of these studies $[19,43]$, though authors also reported that posttreatment and follow-up scores did not differ significantly in any of the groups. One study [50] reported an effect size of $\mathrm{g}=0.31(95 \% \mathrm{CI}=0.08-0.53, p=0.01)$ for self-help CBT compared with control at follow-up, and another study reported maintained rates of clinically significant change from post-treatment [48]. One further study reported sustained treatment gains in treatment group participants for whom follow-up assessments were conducted [57].

Two (out of four) studies of combined treatment reported follow-up; one reported an effect size of $\mathrm{g}=0.37$ $(95 \% \mathrm{CI}=0.02-0.72, p=0.04)$ for stepped-care compared with CAU [54], and the other reported maintenance of gains within the treatment group, but no significant effect of stepped-care compared to CAU due to improvements in the control group at follow-up [55]. Follow-up was not reported in any of the pharmacological treatment studies.

\section{Risk of bias in included studies}

The majority of included studies had an unclear risk of bias for one or more key domains (see Fig. 3 for risk of bias in each study, and Fig. 4 for a summary of risk of bias items across all studies). Interrater agreement between authors ELP and DBF was $85.3 \%$ for risk of bias information. In psychological and combined treatment studies, the risk of performance bias was unclear in most studies, as participants were often not blinded. These studies were also at risk of detection bias due to the use of self-report measures (and unblinded participants) or unblinded outcome assessors. Risk of reporting bias was considered low for studies of psychological or combined treatment, and risk of selection bias was low-to-unclear, with most studies assessed as low risk. Studies of any treatment type 
tended to report equal rates of drop-out across treatment conditions and used intention-to-treat analyses.

For the majority of pharmacological treatment studies, risk of bias was unclear-to-high across domains. All four studies reported inadequate information about random sequence generation and allocation concealment. Three studies had a high risk of bias due to selective outcome reporting, as they presented results visually without reporting outcome statistics (i.e., one or more of the following were missing: means, standard deviations, results of statistical analyses). Furthermore, three of the studies were funded or partially funded by pharmaceutical companies $[46,47,51]$ and for all four studies no conflict of interest statement was included.

\section{Secondary outcomes}

Most included studies ( $n=15,83.3 \%$ of 18$)$ measured depressive symptoms as secondary outcomes, or as combined primary outcomes along with anxiety symptoms. The majority of these $(n=8)$ reported no significant difference in depressive symptoms between control and treatment groups. The seven studies that found a significant treatment effect on depressive symptoms reported effect sizes ranging from $g=0.35$ to 1.00 .

Less than half of the studies $(n=7,38.8 \%$ of 18$)$ included measurements of quality of life. Three studies reported no significant difference in quality of life between groups, and four studies found significant treatment effects ranging from $g=0.31$ to 1.36 .

\section{Discussion}

Our review investigated both psychological and pharmacological treatments for anxiety and explored the effects of treatment provider on psychological treatment effectiveness. Studies of psychological treatment were diverse and could broadly be categorised into two subgroups those that investigated anxiety specifically, and those that investigated common mental disorders (anxiety, depressive, stress, and adjustment disorders).

Meta-analysis demonstrated that for those with primarily anxiety-related difficulties, psychological treatments (predominantly CBT) are effective for reducing anxiety symptoms when provided in primary care. However, the magnitude of this improvement differs depending on who is providing treatment, and is relative to the comparison group. When a specialist provides treatment, large improvements are seen in anxiety symptoms regardless of the type of control group, though the effect is smaller when treatment is compared to other usual treatments than waitlist control. Treatments provided by a non-specialist are also associated with large improvements compared to waitlist control (i.e., no care at all), but were not found to improve anxiety over other usual

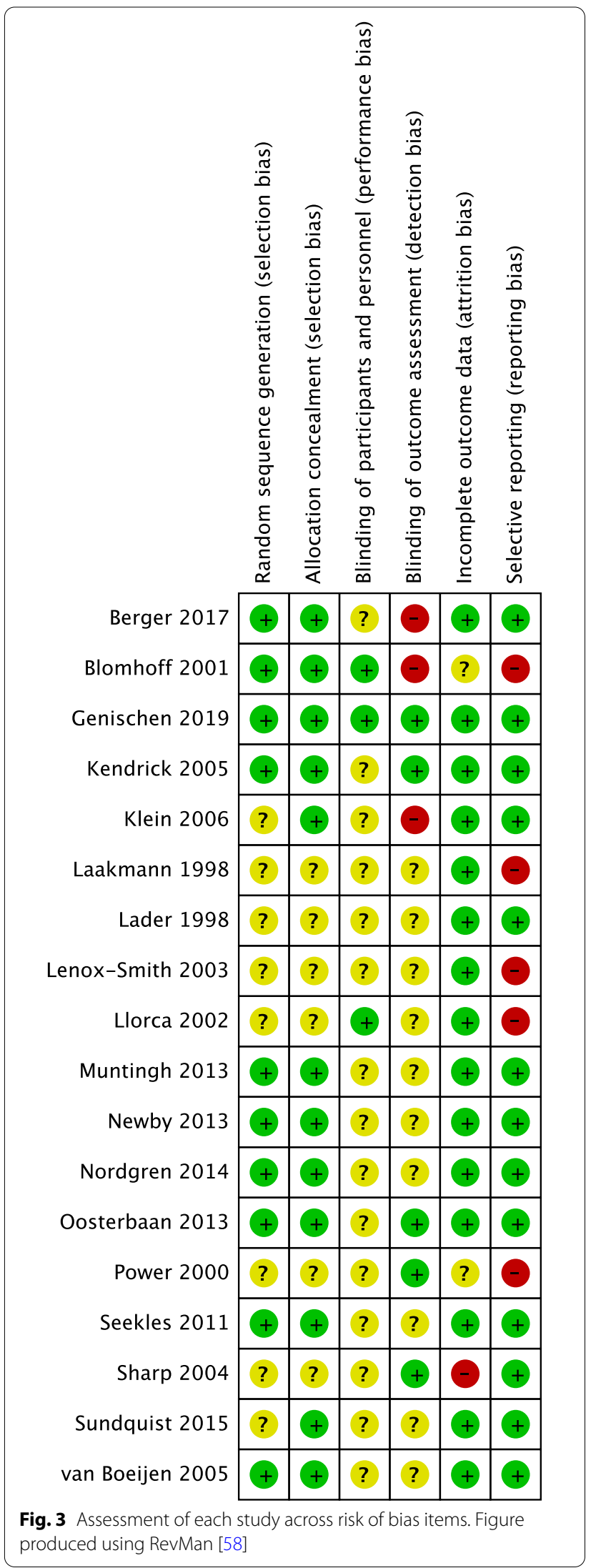




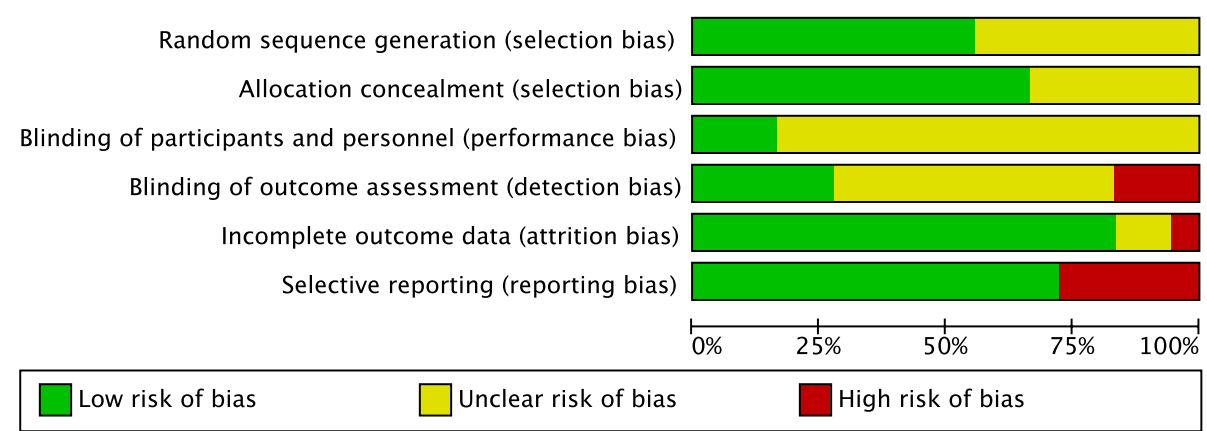

Fig. 4 Assessment of each risk of bias item, presented as proportion of studies with low, unclear, and high risk of bias. Figure produced using RevMan [58]

treatments. These findings are consistent with a previous review of psychological treatment for anxiety in primary care, which demonstrated a superior treatment effect for interventions provided by specialist mental health professionals compared with non-specialists [23]. Previous research has also demonstrated that for both face-toface CBT and computerised CBT, effect sizes are smaller when comparing to CAU (which involves active treatment) than inactive control groups such as waitlist or placebo [20, 23].

Cognitive behaviour therapy is well documented as an effective treatment for anxiety [13, 23], though further research is needed on long-term effectiveness in primary care. In the studies included in our review, CBT was predominantly provided via bibliotherapy or computerised methods, with varying degrees of support from a clinician. The effectiveness of self-help CBT has been demonstrated in other reviews $[20,21]$, and our results provide support for the implementation of these interventions for anxiety in primary care. Computerised CBT has the additional benefit of high fidelity, as interventions can be delivered exactly as designed. This is in contrast to faceto-face therapy where fidelity is impacted by experience and training of the provider and their adherence to treatment manuals, which may be particularly relevant for non-specialist treatment providers [13].

The results for longer-term follow-up in psychological treatment studies included in our review were mixed. However, most reported treatment gains were maintained within the treatment group, and were superior to gains seen in control group participants who received other usual treatments. Limited data on long-term follow-up is a limitation in the field, though studies not specific to primary care settings have found that the effect of psychological treatment for anxiety tends to be well maintained at follow-up $[59,60]$.

The studies investigating treatment for common mental disorders were summarised using narrative synthesis as there were too few studies to conduct meta-analysis. The pattern of results across these studies was similar to that of the studies on anxiety only; psychological treatments did not produce a significant effect compared with CAU control groups, though large effects of treatment were seen when compared to waitlist control.

Only a small number of included studies involved pharmacological treatment, and only two $[42,51]$ involved current first-line agents for anxiety (sertraline and venlafaxine) [12]. Both medications produced small, superior effects compared to placebo, indicating they are effective for reducing anxiety symptoms in primary care. Across an additional three studies, hydroxyzine also produced small to moderate effects, while buspirone and benzodiazepines were not found to reduce anxiety compared with placebo. However, hydroxyzine and buspirone are not considered first-line agents for anxiety, and benzodiazepines are only recommended in specific conditions such as during the initiation phase of an SSRI [61]. Furthermore, the majority of pharmacological treatment studies were funded by pharmaceutical companies and had a high risk of bias due to selective outcome reporting, questioning the validity of these results. Overall, we did not find a strong body of research documenting the use of pharmacological treatments in primary care. This was true irrespective of the exclusion of studies from countries without universal healthcare, as only one additional study of medication (an SSRI) would have been included if not for this restriction.

None of the included studies of pharmacological treatment reported on longer-term follow-up, so we were not able to investigate the effectiveness of these medications beyond the acute treatment phase. Previous research has demonstrated that the risk of relapse is high when pharmacological interventions are discontinued following acute treatment, and it is therefore advised that treatment continue for between six and 24-months after remission [62]. Given pharmacological interventions are 
the dominant treatment strategy provided in primary care, further research is needed to determine the effectiveness of these treatments in this setting.

The combined use of medication and psychological therapy was directly investigated in only one study [42]. This demonstrated combined treatment was effective in comparison to control but no more effective than either treatment alone. Although combined treatment is commonly used in practice, there is limited evidence to indicate this leads to better outcomes [13]. Stepped care interventions, including both pharmacological and psychological treatment steps, appear effective for treating anxiety based on the three studies included in our review. Results from these studies are consistent with the emerging body of evidence for collaborative stepped care in primary care, with small to moderate effect sizes found in a previous review [63].

\section{Limitations}

Our review had several limitations. Studies were heterogeneous and meta-analytic results for the effects of psychological treatment should be interpreted with caution. Several factors may have contributed to heterogeneity in this review. For example, across the included studies there was a mixture of self-report and clinician assessed measures, and treatment was provided using a variety of modalities (e.g., online, individual face-to-face, group). Likewise, multiple anxiety disorders were investigated both within and between studies, and different disorders may have responded differently to the treatments used. Unfortunately, additional moderators, including the planned investigation of treatment modality, were not able to be explored due to the small number of included studies. The decision to pool studies using meta-analysis is based on both statistical and theoretical considerations. It is important to note the heterogeneous nature of primary care, and diversity among included studies can be considered a reflection of the real-world treatment provided in this setting. Combining studies of diverse interventions may not provide meaningful information about the individual effects of each intervention, but can be useful in answering broader questions (e.g., summarising the average effect of a class of drugs by combining studies of different drugs within that class) [31]. Although heterogeneity limits the strength of conclusions that can be drawn from our meta-analytic results, we believe our findings are useful in contributing to the broader question of how well psychological interventions work for anxiety in primary care.

Another limitation of our review is that the effect of psychological treatments compared with CAU is difficult to interpret, as CAU was poorly described in the included studies. Control group participants could receive medication, other psychological treatments, general advice, or no treatment at all, and most studies did not report the rates of different care. However, studies reported that at least half of control group participants received some form of active intervention, including referral for specialist mental health care and antidepressant medication. This may have reduced the apparent effectiveness of treatments provided by non-specialists in particular, as participants in the control condition may have received a higher intensity treatment such as specialist psychological treatment, medication, or both.

As with all systematic reviews, our search strategy and inclusion criteria may have excluded relevant studies of treatment for anxiety in primary care. This is particularly true of studies conducted in countries without universal healthcare systems (most notably, the USA), and studies that were published in languages other than English. We also identified very few studies of primary care specific pharmacological treatment, and may have identified further studies if we had searched additional biomedical databases (e.g., Embase). Unfortunately, we did not have access to Embase for this review.

Despite attempts to maximise identification of studies with non-specialist treatment providers, we identified relatively few studies of psychological treatments provided by GPs. Combined with the limited number of pharmacological treatment studies, the body of evidence identified is inconsistent with the real-world treatment of anxiety disorders in primary care $[6,11]$ and limits our ability to describe the effectiveness of this treatment. The generalisability of our findings to low-income countries and high-income countries without universal health care is also limited. Finally, only one study was identified that directly compared medication and psychological treatments in primary care, making it difficult to comment on the relative effectiveness of the two. Other reviews have noted the lack of comparison between psychological and pharmacological treatments as a serious limitation in the field, particularly in the case of computerised CBT programs versus medication [20].

\section{Implications for clinical practice}

Despite the limitations, our review has several important implications for primary care. Results support previous research in this area, demonstrating that CBT-based psychological treatments for anxiety are effective, and that specialist treatment (i.e., provided by a psychologist or clinical psychologist) is preferable [23]. Our results also extend upon previous findings by providing information about treatment delivered by non-specialists, which is important given that access to specialists is not always possible in primary care. Although we did not find that psychological treatment provided by non-specialists is superior to other 
usual treatments, we also did not find it to be inferior. This indicates that non-specialist psychological treatment may be at least as good as other usual treatments, and an appropriate option for consumers. Additionally, our results demonstrated that non-specialist treatment is associated with significant and large improvements in anxiety compared with no treatment at all.

Although pharmacological treatments are effective for anxiety generally [61] and have advantages in terms of cost and ease of access, we did not find strong evidence for their use in primary care due to a small number of studies and high-risk of bias among those studies. Medications for anxiety disorders carry side effects [64], and benzodiazepines, which remain commonly prescribed despite no longer being a recommended first-line treatment $[24,25]$, carry risks of both physiological and psychological dependence. Furthermore, benzodiazepines may in fact prolong anxiety symptoms if used alone due to their use as a safety behaviour and potential to impair fear extinction [65, 66]. This may be particularly true when physiological anxiety sensations themselves are the feared stimuli (e.g., in panic disorder), and exposure to these symptoms is avoided through the use of benzodiazepines.

We therefore recommend that pharmacological treatments be used with caution in primary care until further research is conducted, and that CBT-based psychological treatments, including those provided online and via self-help, be offered as first-line treatments for anxiety disorders in this setting. This treatment should be provided by a specialist such as a psychologist or clinical psychologist if available and affordable for the consumer. However, non-specialists should still offer psychological treatment if specialist treatment is not possible.

\section{Conclusions}

Overall, our review demonstrated that, in countries with universal healthcare, a greater alignment of research and practice is needed to more effectively manage anxiety disorders. Additional research is needed to investigate the use of pharmacological treatments in primary care and to determine their relative effectiveness when compared with psychological interventions in this setting. Future research on psychological treatments should aim to more closely mirror the treatment that is delivered in real-world primary care settings (i.e., in terms of treatment provider). This research should be conducted alongside implementation science involving both provider and consumer perspectives, that explores barriers to the delivery of psychological treatments for anxiety in primary care.

\section{Abbreviations}

BAI: Beck anxiety inventory; CAU: Care as usual; CBT: Cognitive behaviour therapy; DASS: Depression anxiety stress scale; DSM: Diagnostic and statistical manual of mental disorders; GAD: Generalised anxiety disorder; GP: General practitioner; ICD: International classification of diseases; OCD: Obsessive compulsive disorder; PTSD: Post-traumatic stress disorder; SNRI: Serotonin noradrenaline reuptake inhibitors; SSRI: Selective serotonin reuptake inhibitor.

\section{Supplementary Information}

The online version contains supplementary material available at https://doi. org/10.1186/s12875-021-01445-5.

Additional file 1. Additional Table. Full Search Strategy. Full search strategy used for all databases.

\section{Acknowledgements}

The first author conducted this review under the supervision of the second, third, and last authors in partial fulfilment of a Doctor of Philosophy in Clinical Psychology at the Australian National University (ANU). We also thank Professor Philip Batterham for his contributions to this review.

\section{Authors' contributions}

ELP, MB, DBF, and MK devised the concept and design of the study. ELP and $\mathrm{TH}$ assessed studies for eligibility. Data were extracted from all articles by ELP and either TH or DBF. ELP conducted the analyses, and ELP, MB, and DBF interpreted data. ELP drafted the article, and all authors revised it critically for content and approved the version to be published.

\section{Funding}

This research received no specific grant from any funding agency, commercial or not-for-profit sectors. ELP was supported by an Australian Government Research Training Program (AGRTP) Stipend Scholarship for the duration of the review. MB is supported by a Medical Research Future Fund (MRFF) Translating Research into Practice (TRIP) Fellowship number MRF1150698, which is unrelated to the submitted work. These funders had no role in study design, data collection, data analysis, data interpretation, or writing of the report.

\section{Availability of data and materials}

All data generated or analysed during this study are included in this published article, its additional files, and the published articles included in this review.

\section{Declarations}

Ethics approval and consent to participate Ethics approval was not require for this study.

\section{Consent for publication}

Not applicable.

\section{Competing interests}

ELP is paid by Marathon Health for provision of psychological therapy services and runs a private practice as a psychologist. No other relationships or activities with potential to bias the work are identified.

\section{Author details}

${ }^{1}$ Research School of Psychology, Australian National University, Canberra, ACT 2601, Australia. ${ }^{2}$ Centre for Mental Health Research, Australian National University, Canberra, Australia. ${ }^{3}$ College of Education, Psychology and Social Work, Flinders University, Adelaide, Australia.

Received: 27 September 2020 Accepted: 26 April 2021

Published online: 15 May 2021 


\section{References}

1. Baxter AJ, Scott KM, Vos T, Whiteford HA. Global prevalence of anxiety disorders: a systematic review and meta-regression. Psychol Med. 2013;43(5):897-910.

2. Kessler RC. The global burden of anxiety and mood disorders: putting the European Study of the Epidemiology of Mental Disorders (ESEMeD) findings into perspective. J Clin Psychiatry. 2007;68(Suppl 2):10-9.

3. Baxter AJ, Vos T, Scott KM, Ferrari AJ. The global burden of anxiety disorders in 2010. Psychol Med. 2014:44(11):2363-74.

4. Burgess PM, Pirkis JE, Slade TN, Johnston AK, Meadows GN, Gunn JM. Service use for mental health problems: findings from the 2007 National Survey of Mental Health and Wellbeing. Aust N Z J Psychiatry. 2009;43(7):615-23.

5. Bijl RV, Ravelli A. Psychiatric morbidity, service use, and need for care in the general population: results of the Netherlands Mental Health Survey and Incidence Study. Am J Public Health. 2000:90(4):602-7.

6. Wang PS, Aguilar-Gaxiola S, Alonso J, Angermeyer MC, Borges G, Brome EJ, Bruffaerts R, de Girolamo G, de Graaf R, Gureje O, et al. Use of mental health services for anxiety, mood, and substance disorders in 17 countries in the WHO world mental health surveys. Lancet (London, England). 2007;370(9590):841-50

7. World Health Organization. The WHO special initiative for mental health (2019-2023): Universal health coverage for mental health. World Health Organization; 2019. https://apps.who.int/iris/handle/10665/310981.

8. Chapdelaine A, Carrier J-D, Fournier L, Duhoux A, Roberge P. Treatment adequacy for social anxiety disorder in primary care patients. PLOS ONE. 2018;13(11):e0206357-e0206357.

9. Harris MG, Hobbs MJ, Burgess PM, Pirkis JE, Diminic S, Siskind DJ, Andrews $\mathrm{G}$, Whiteford HA. Frequency and quality of mental health treatment for affective and anxiety disorders among Australian adults. Med J Aust. 2015:202(4):185-9.

10. Wittchen HU. Generalized anxiety disorder: prevalence, burden, and cost to society. Depress Anxiety. 2002;16(4):162-71.

11. Britt H, Miller GC, Henderson J, Bayram C, Harrison C, Valenti L, Pan Y, Charles J, Pollack AJ, Wong C, et al. General practice activity in Australia 2015-16. Sydney: Sydney University Press; 2016.

12. National Institute for Health and Care Excellence. Anxiety disorders: quality standard. London: Author; 2014.

13. Andrews G, Bell C, Boyce P, Gale C, Lampe L, Marwat O, Rapee R, Wilkins G. Royal Australian and New Zealand College of Psychiatrists clinical practice guidelines for the treatment of panic disorder, social anxiety disorder and generalised anxiety disorder. Aust N Z J Psychiatry. 2018;52(12):1109-72.

14. National Institute for Health and Care Excellence. Generalised anxiety disorder and panic disorder in adults: management. London: Author; 2011.

15. National Institute for Health and Care Excellence. Social anxiety disorder: recognition, assessment and treatment. London: Author; 2013.

16. van Schaik DJF, Klijn AFJ, van Hout HPJ, van Marwijk HWJ, Beekman ATF, de Haan M, van Dyck R. Patients' preferences in the treatment of depressive disorder in primary care. Gen Hosp Psychiatry. 2004;26(3):184-9.

17. Mohlman J. A community based survey of older adults' preferences for treatment of anxiety. Psychol Aging. 2012;27:1182-90.

18. Richards JC, Ryan P, Mccabe MP, Groom G, Hickie IB. Barriers to the effective management of depression in general practice. Aust N Z J Psychiatry. 2004;38(10):795-803.

19. van Boeijen CA, van Oppen $P$, van Balkom AJLM, Visser S, Kempe PT, Blankenstein N, van Dyck R. Treatment of anxiety disorders in primary care practice: a randomised controlled trial. Br J Gen Pract. 2005;55(519):763-9.

20. Andrews G, Basu A, Cuijpers P, Craske MG, McEvoy P, English CL, Newby JM. Computer therapy for the anxiety and depression disorders is effective, acceptable and practical health care: an updated meta-analysis. J Anxiety Disord. 2018:55:70-8.

21. Andrews G, Cuijpers P, Craske MG, McEvoy P, Titov N. Computer therapy for the anxiety and depressive disorders is effective, acceptable and practical health care: a meta-analysis. PLoS ONE. 2010;5(10):e13196.

22. Patel V, Saxena S. Achieving universal health coverage for mental disorders. BMJ. 2019;366:14516.

23. Seekles W, Cuijpers P, Kok R, Beekman A, van Marwijk H, van Straten A. Psychological treatment of anxiety in primary care: a meta-analysis. Psychol Med. 2013;43(2):351-61.
24. Sonnenberg CM, Bierman EJ, Deeg DJ, Comijs HC, van Tilburg W, Beekman ATF. Ten-year trends in benzodiazepine use in the Dutch population. Soc Psychiatry Psychiatr Epidemiol. 2012;47(2):293-301.

25. Stephenson CP, Karanges E, McGregor IS. Trends in the utilisation of psychotropic medications in Australia from 2000 to 2011. Aust N Z J Psychiatry. 2013;47(1):74-87.

26. Hedges LV. Distribution theory for glass's estimator of effect size and related estimators. J Educ Behav Stat. 1981;6(2):107-28.

27. Hedges LV, Olkin I. Statistical methods for meta-analysis. Orlando: Academic Press; 1985.

28. Viechtbauer W. Conducting meta-analyses in $\mathrm{R}$ with the metafor package J Stat Softw. 2010;36(3):1-48.

29. Gleser LJ, Olkin I. Stochastically dependent effect sizes. In: Cooper H, Hedges LV, Valentine JC, editors. The handbook of research synthesis and meta-analysis. 2nd ed. New York: Russell Sage Foundation; 2009. p. 357-76.

30. Berkey CS, Hoaglin DC, Antczak-Bouckoms A, Mosteller F, Colditz GA. Meta-analysis of multiple outcomes by regression with random effects. Stat Med. 1998;17(22):2537-50.

31. Deeks JJ, Higgins JPT, Altman DG. Chapter 10: analysing data and undertaking meta-analyses. In: Higgins JPT, Thomas J, Chandler J, Cumpston M, Li T, Page MJ, editors. Cochrane handbook for systematic reviews of interventions version 61 (updated September 2020). Welch: The Cochrane Collaboration; 2020.

32. Egger M, Smith GD, Schneider M, Minder C. Bias in meta-analysis detected by a simple, graphical test. BMJ. 1997;315(7109):629-34

33. Sterne JA, Egger M. Regression methods to detect publication and other bias in meta-analysis. In: Rothstein HR, editor. Publication bias in metaanalysis: Prevention, assessment and adjustments. Sutton AJ: M B; 2005. p. $99-110$.

34. Viechtbauer W, Cheung MW-L. Outlier and influence diagnostics for meta-analysis. Res Synth Methods. 2010;1 (2):112-25.

35. Cook RD. Influential observations in linear regression. J Am Stat Assoc. 1979;74(365):169-74

36. Cook RD. Detection of influential observation in linear regression. Technometrics. 1977:19(1):15-8.

37. Higgins JPT, Green S, editors. Cochrane handbook for systematic reviews of interventions version 5.1.0 (updated March 2011). The Cochrane Collaboration; 2011. www.handbook.cochrane.org

38. Christensen H, Pallister E, Smale S, Hickie IB, Calear AL. Community-based prevention programs for anxiety and depression in youth: a systematic review. J Primary Prevent. 2010;31(3):139-70.

39. Roy-Byrne P, Craske MG, Sullivan G, Rose RD, Edlund MJ, Lang AJ, Bystritsky A, Shaw Welch S, Chavira DA, Golinelli D, et al. Delivery of evidence-based treatment for multiple anxiety disorders in primary care: A randomized controlled trial. J Am Med Assoc. 2010;303(19):1921-8.

40. Seekles W, van Straten A, Beekman A, van Marwijk H, Cuijpers P. Effectiveness of guided self-help for depression and anxiety disorders in primary care: a pragmatic randomized controlled trial. Psychiatry Res. 2011;187(1-2):113-20.

41. Seekles W, van Straten A, Beekman A, van Marwijk H, Cuijpers P. Stepped care treatment for depression and anxiety in primary care. a randomized controlled trial. Trials. 2011:12:171.

42. Blomhoff S, Haug TT, Hellström K, Holme I, Humble M, Madsbu HP, Wold JE. Randomised controlled general practice trial of sertraline, exposure therapy and combined treatment in generalised social phobia. $\mathrm{Br} J$ Psychiatry. 2001;179(1):23-30.

43. Kendrick T, Simons L, Mynors-Wallis L, Gray A, Lathlean J, Pickering R, Harris S, Rivero-Arias O, Gerard K, Thompson C. A trial of problem-solving by community mental health nurses for anxiety, depression and life difficulties among general practice patients The CPN-GP study. Health Technol Assess. 2005:9(37):1-104.

44. Klein B, Richards JC, Austin DW. Efficacy of internet therapy for panic disorder. J Behav Ther Exp Psychiatry. 2006;37(3):213-38.

45. Laakmann G, Schüle C, Lorkowski G, Baghai T, Kuhn K, Ehrentraut S. Buspirone and lorazepam in the treatment of generalized anxiety disorder in outpatients. Psychopharmacology. 1998;136(4):357-66.

46. Lader M, Scotto JC. A multicentre double-blind comparison of hydroxyzine, buspirone and placebo in patients with generalized anxiety disorder. Psychopharmacology. 1998;139(4):402-6. 
47. Llorca PM, Spadone C, Sol O, Danniau A, Bougerol T, Corruble E, Faruch M, Macher JP, Sermet E, Servant D. Efficacy and safety of hydroxyzine in the treatment of generalized anxiety disorder: A 3-month double-blind study. J Clin Psychiatry. 2002;63(11):1020-7.

48. Power KG, Sharp DM, Swanson V, Simpson R. Therapist contact in cognitive behaviour therapy for panic disorder and agoraphobia in primary care. Clin Psychol Psychother. 2000;7(1):37-46.

49. Sharp DM, Power KG, Swanson V. A comparison of the efficacy and acceptability of group versus individual cognitive behaviour therapy in the treatment of panic disorder and agoraphobia in primary care. Clin Psychol Psychother. 2004;11(2):73-82.

50. Gensichen J, Hiller TS, Breitbart J, Brettschneider C, Teismann T, Schumacher U, Lukaschek K, Schelle M, Schneider N, Sommer M, et al. Panic disorder in primary care. Dtsch Arztebl Int. 2019;116(10):159-66.

51. Lenox-Smith AJ, Reynolds A. A double-blind, randomised, placebo controlled study of venlafaxine $\mathrm{XL}$ in patients with generalised anxiety disorder in primary care. Br J Gen Pract. 2003;53(495):772-7.

52. Sundquist J, Lilja A, Palmer K, Memon AA, Wang X, Johansson LM, Sundquist K. Mindfulness group therapy in primary care patients with depression, anxiety and stress and adjustment disorders: randomised controlled trial. Br J Psychiatry. 2015;206(2):128-35.

53. Newby JM, Mackenzie A, Williams AD, Mclntyre K, Watts S, Wong N, Andrews $\mathrm{G}$. Internet cognitive behavioural therapy for mixed anxiety and depression: a randomized controlled trial and evidence of effectiveness in primary care. Psychol Med. 2013;43(12):2635-48.

54. Muntingh ADT, Van Der Feltz-Cornelis C, Van Marwijk H, Spinhoven P, Assendelft W, De Waal M, Adèr H, Van Balkom A. Effectiveness of collaborative stepped care for anxiety disorders in primary care: a pragmatic cluster randomised controlled trial. Psychother Psychosom. 2014;83(1):37-44.

55. Oosterbaan DB, Verbraak MJPM, Terluin B, Hoogendoorn AW, Peyrot WJ, Muntingh A, Van Balkom AJLM. Collaborative stepped care v. care as usual for common mental disorders: 8-month, cluster randomised controlled trial. Br J Psychiatry. 2013;203(2):132-139.

56. Nordgren LB, Hedman E, Etienne J, Bodin J, Kadowaki A, Eriksson S, Lindkvist E, Andersson G, Carlbring P. Effectiveness and cost-effectiveness of individually tailored Internet-delivered cognitive behavior therapy for anxiety disorders in a primary care population: a randomized controlled trial. Behav Res Ther. 2014;59:1-11.

57. Berger T, Urech A, Krieger T, Stolz T, Schulz A, Vincent A, Moser CT, Moritz $\mathrm{S}$, Meyer B. Effects of a transdiagnostic unguided Internet intervention ('velibra') for anxiety disorders in primary care: Results of a randomized controlled trial. Psychol Med. 2017;47(1):67-80.

58. Review Manager (RevMan) [Computer Program]. Version 5.3. Copenhagen: The Nordic Cochrane Centre, The Cochrane Collaboration; 2014.

59. Mörtberg E, Clark DM, Bejerot S. Intensive group cognitive therapy and individual cognitive therapy for social phobia: sustained improvement at 5-year follow-up. J Anxiety Disord. 2011;25(8):994-1000.

60. van Dis EAM, van Veen SC, Hagenaars MA, Batelaan NM, Bockting CLH, van den Heuvel RM, Cuijpers P, Engelhard IM. Long-term outcomes of cognitive behavioral therapy for anxiety-related disorders: a systematic review and meta-analysis. JAMA Psychiat. 2020;77(3):265-73.

61. Ravindran $L N$, Stein MB. The pharmacologic treatment of anxiety disorders: A review of progress. J Clin Psychiatry. 2010;71(7):839-54.

62. Bandelow B, Sher L, Bunevicius R, Hollander E, Kasper S, Zohar J, Möller $\mathrm{H}$-J. Guidelines for the pharmacological treatment of anxiety disorders, obsessive-compulsive disorder and posttraumatic stress disorder in primary care. Int J Psychiatry Clin Pract. 2012;16(2):77-84.

63. Muntingh ADT, van der Feltz-Cornelis CM, van Marwijk HWJ, Spinhoven P, van Balkom AJLM. Collaborative care for anxiety disorders in primary care: a systematic review and meta-analysis. BMC Fam Pract. 2016;17(1):62.

64. Wang S-M, Han C, Bahk W-M, Lee S-J, Patkar AA, Masand PS, Pae C-U. Addressing the side effects of contemporary antidepressant drugs: a comprehensive review. Chonnam Med J. 2018;54(2):101-12.

65. Hart G, Panayi MC, Harris JA, Westbrook RF. Benzodiazepine treatment can impair or spare extinction, depending on when it is given. Behav Res Ther. 2014;56:22-9.

66. Westra HA, Stewart SH, Conrad BE. Naturalistic manner of benzodiazepine use and cognitive behavioral therapy outcome in panic disorder with agoraphobia. J Anxiety Disord. 2002;16(3):233-46.

\section{Publisher's Note}

Springer Nature remains neutral with regard to jurisdictional claims in published maps and institutional affiliations.
Ready to submit your research? Choose BMC and benefit from:

- fast, convenient online submission

- thorough peer review by experienced researchers in your field

- rapid publication on acceptance

- support for research data, including large and complex data types

- gold Open Access which fosters wider collaboration and increased citations

- maximum visibility for your research: over 100M website views per year

At BMC, research is always in progress.

Learn more biomedcentral.com/submissions 